\title{
Field Application of Cable Tension Estimation Technique Using the h-SI Method
}

\author{
Myung-Hyun Noh ${ }^{1}$ and WooYoung Jung ${ }^{2}$ \\ ${ }^{1}$ Steel Solution Center, POSCO, 100 Songdogwahak-ro, Yeonsu-gu, Incheon 406-840, Republic of Korea \\ ${ }^{2}$ Department of Civil Engineering, Gangneung-Wonju National University, Gangneung 210-702, Republic of Korea \\ Correspondence should be addressed to WooYoung Jung; woojung@gwnu.ac.kr
}

Received 31 July 2014; Accepted 13 August 2014

Academic Editor: Sang-Youl Lee

Copyright (c) 2015 M.-H. Noh and W. Jung. This is an open access article distributed under the Creative Commons Attribution License, which permits unrestricted use, distribution, and reproduction in any medium, provided the original work is properly cited.

\begin{abstract}
This paper investigates field applicability of a new system identification technique of estimating tensile force for a cable of long span bridges. The newly proposed h-SI method using the combination of the sensitivity updating algorithm and the advanced hybrid microgenetic algorithm can allow not only avoiding the trap of local minimum at initial searching stage but also finding the optimal solution in terms of better numerical efficiency than existing methods. First, this paper overviews the procedure of tension estimation through a theoretical formulation. Secondly, the validity of the proposed technique is numerically examined using a set of dynamic data obtained from benchmark numerical samples considering the effect of sag extensibility and bending stiffness of a sag-cable system. Finally, the feasibility of the proposed method is investigated through actual field data extracted from a cable-stayed Seohae Bridge. The test results show that the existing methods require precise initial data in advance but the proposed method is not affected by such initial information. In particular, the proposed method can improve accuracy and convergence rate toward final values. Consequently, the proposed method can be more effective than existing methods in terms of characterizing the tensile force variation for cable structures.
\end{abstract}

\section{Introduction}

Recently, the number of cable-stayed structures built in countries around the world is increasing. The cable-stayed structures deteriorate due to a variety of reasons such as overloading, aging, manufacturing imperfection, and climate conditions. Localized damage in materials may make deleterious contributions to the tensile force of the cable system. Therefore, it is necessary to estimate the tension of the cables to ensure structural safety during the construction phase and maintenance work after completion.

The tensile force of the cable system can be characterized by means of a condition assessment technology based on a variety of disciplines. Among the many nondestructive evaluation (NDE) methods, this study is focused on the use of dynamic response in detecting tensile forces in the cable structure system. Of the many condition assessment techniques for cable-stayed structures available today, system identification methods are based on detecting the changes in static or dynamic behavior of a cable [1-3]. The tensile force of cables could be inversely determined by using mathematical models based on the taut string theory or axially loaded beam theory from natural frequency data. These works, based on simplified analytical approaches, have limited capabilities in dealing with complex problems, primarily due to their limitations in handling real cable shapes in the analysis. The differences between the real structural system and mathematical model for various cables make deleterious contributions to the accurate detection of the tensile force [4]. In addition, Bati and Tonietti [5] presented the significance of the flexural rigidity of cables in determining tensile forces. They reported that the tensile force induced from the simplified string model, which does not consider flexural rigidity, is not reliable for most cases. The difference becomes more dramatic for cases of the geometrical shape of cables, construction tolerances, or support conditions. Blasi and Sorace [6] proposed a method for detecting tensile forces by combining static and dynamic identification techniques, which reflect 
the characteristics of the tie-rod structural system. Park et al. [7] carried out the identification of the tensile force in high-tension bars using modal sensitivities. Park et al. [8] developed a system identification technique to determine the tensile force and various rigidities of cables simultaneously by using the finite element method and sensitivity equation. They proved the proposed method through lab-scale and field test.

These works, based on the local optimization algorithm (LOA), have limited capabilities in dealing with complex problems, primarily due to their limitations in handling assumed initial conditions in the analysis. They have several limitations, such as divergence and instability problems, during numerical calculations. In particular, the trap problem of false minimum is frequently observed for large and complicated structures. In recent years, global optimization algorithms (GOA), such as neural networks, genetic algorithms (GAs), and simulated annealing methods have been developed and promisingly applied to the field of structural identification. Among them, GAs attracted our attention because not a great deal of data was needed in advance. This is an advantage over natural frequency-based neural network methods that require priori-knowledge of both the modal frequencies and the modal shapes to train the neural network and to detect the structural damage. Lee and Wooh [9] presented a microgenetic algorithm that is able to identify the location and extent of damage in plate-type structures using only the frequency information. Noh et al. [10] suggested a differential evolutionary algorithm to determine external tendon forces and Rayleigh damping coefficients.

Despite the broad spectrum of applications, the conventional GAs usually require a large number of iterations and thus high computational cost. To solve an inverse problem using a GA, it is necessary to carry out iterative forward computations for each individual. Noh and Lee [11] developed a hybrid genetic algorithm (h-GA) to reduce the iterations by using the organic-hybridization technique. For a cable-stayed structure, global optimization algorithms such as GAs and an efficient sensitivity method could serve a dominant role in improving the convergence. Noh and $\mathrm{Hu}$ [12] developed a new algorithm using the advantages of both the LOA and GOA. They adopted the posthybridization method where the GOA provides the initial values for the LOA. The most significant characteristic of this method is that the hybrid microgentic algorithm (h-GA), which is a GOA, helps solve the local convergence problem due to incorrect initial value settings, by using the sensitivity-updating algorithm (SUA), which is a LOA, to improve the precision and convergence to the final value. However, the validity of the new method has been restrictively verified through numerical and laboratoryscale studies from computation efficiency point of view. Therefore, it will be also necessary to prove the concept from further experimental studies for real cable-stayed long-span bridges.

In this paper, we will focus on the practical applications of the proposed method to cable-stayed long-span bridge suggested in the study of Noh and $\mathrm{Hu}$ [12]. First, the process of tension estimation using the h-SI method is reformulated in a general form theoretically. Secondly, the validity of the technique is numerically verified using a set of dynamic data obtained from a simulation of benchmark numerical samples considering the effect of sag-extensibility and bending stiffness of a sag-cable system. Finally, the feasibility of the proposed method is investigated through field data extracted from a cable-stayed Seohae Bridge, and its applicability is verified.

\section{Theoretical Background for Tension Estimation Method Using the h-SI}

Noh and Hu [12] developed the hybrid system identification method using the combined effects of the hybrid microgentic algorithms (h-GA) and the sensitivity equation for the tension estimation of cable structures. Figure 1 illustrates the hybrid procedure used in this study. The hybrid system identification (h-SI) algorithm consists of h-GA in GOA and the sensitivity-updating algorithm in LOA. It also uses the posthybridization procedure to avoid the local convergence, in which the GOA provides the initial value to LOA.

In the process of tension estimation using the h-SI method based on the finite element model, the tensile force to be identified is a component of an identification vector of the following form:

$$
\mathbf{x}=\left[\begin{array}{llllll}
x^{1} & x^{2} & \cdots & x^{j} & \cdots & x^{p}
\end{array}\right]^{\mathrm{T}},
$$

where $x^{j}(j=1, \ldots, p)$ denotes the component of the identification vector and $p$ denotes the number of identification parameters. The tension of a cable should be a major component of the identification vector, and material and/or geometrical properties of the cable can also be other components.

First, to explain a new reproduction process of the h-GA in GOA different compared to the existing GA algorithm, the reproduction process for mating pools is formulated, assuming the $k$ th generation ${ }^{k} \mathbf{P}$ consisting of $N$ members. Consider

$$
{ }^{k} \mathbf{P}=\left[\begin{array}{lllllll}
{ }^{k} \boldsymbol{\chi}_{1} & { }^{k} \boldsymbol{\chi}_{2} & \cdots & { }^{k} \boldsymbol{\chi}_{i} & \cdots & { }^{k} \boldsymbol{\chi}_{N}
\end{array}\right]^{\mathrm{T}},
$$

where ${ }^{k} \chi_{i}(i=1, \ldots, N)$ denotes the $i$ th individual for every $k$ th generation, is composed of a binary combination of identification parameters for the estimation of the tension, and can be expressed as

$$
{ }^{k} \chi_{i}=\left(\begin{array}{llllllll}
{ }^{k} & \chi_{i}^{1} & { }^{k} & \chi_{i}^{2} & \cdots & { }^{k} \chi_{i}^{j} & \cdots & \chi_{i}^{m}
\end{array}\right),
$$

where ${ }^{k} \chi_{i}^{j}(j=1, \ldots, m)$ denotes a component of the binary string vector for the $i$ th individual of the $k$ th generation, which consists of either 0 or 1 , and $m$ denotes the number of total bits assigned to the string vector composed of the combination of identification.

Next, for the reproduction process, the string vector of (3) can be decoded as

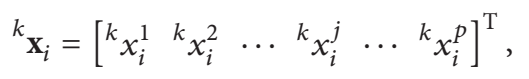




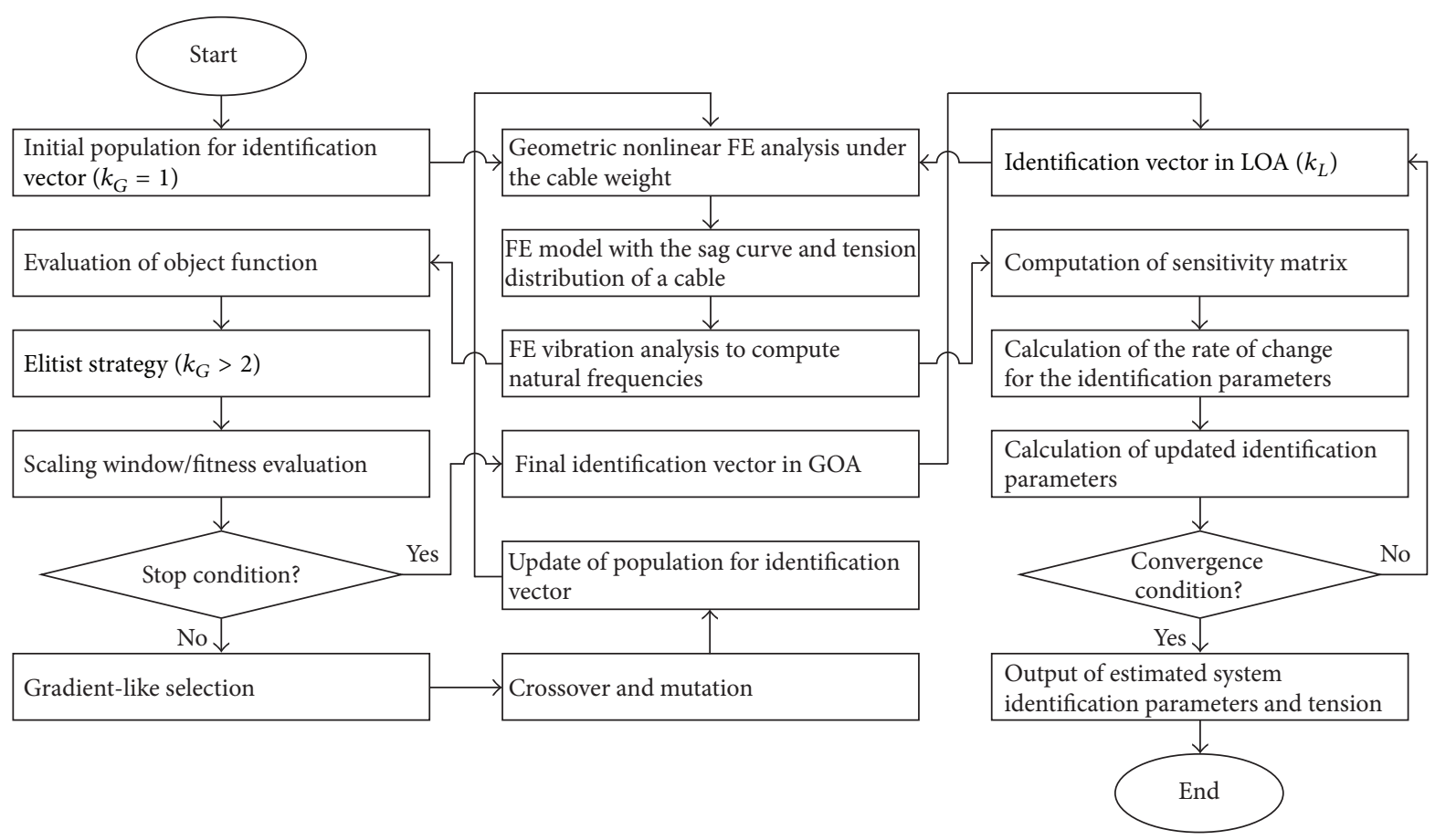

FIgURE 1: A schematic representation of the h-SI method.

where ${ }^{k} x_{i}^{j}$ denotes the $j$ th identification parameter for $i$ th individual in the $k$ th generation. The components of the row vectors are real numbers. Using those row vectors, an identification matrix with $(N \times p)$ size can be defined as

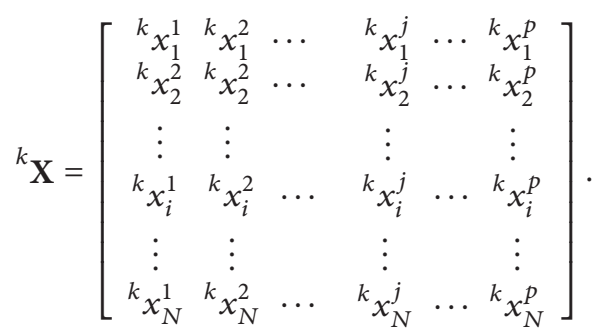

To identify the tension of cables accurately, h-GA should be combined with the nonlinear finite element model that can reflect the properties of cables in the present state. That is, the finite element model parameters that can be sensitive to changes in tension should be determined as the identification parameters in the system identification procedure. In this study, natural frequencies are produced from the finite element model, which are considered the deflection curve and tensile force distribution of cables. The produced frequencies are used for determining the object function $\left({ }^{k} \Xi_{i}\right)$ and fitness values $\left({ }^{k} f_{i}\right)$ as follows:

$$
\begin{gathered}
{ }^{k} \Xi_{i}\left({ }^{k} \mathbf{x}_{i}\right)=\sum_{j=1}^{q}\left\|\omega_{j}{ }^{*}-{ }^{k} \omega_{j}\left({ }^{k} \mathbf{x}_{i}\right)\right\| \\
{ }^{k} f_{i}\left({ }^{k} \mathbf{x}_{i}\right)=-{ }^{k} \Xi_{i}\left({ }^{k} \mathbf{x}_{i}\right)-{ }^{k-1} \gamma(>0),
\end{gathered}
$$

where $\omega_{j}^{*}$ denotes the natural frequency data measured from real cable structures and ${ }^{k} \omega_{j}$ denotes the produced natural frequency data calculated from the finite element vibration model. Then, the best fitness function can be prescribed by finding the minimum of the ${ }^{k} f_{i}\left({ }^{k} \mathbf{x}_{i}\right)$ among the total individuals in a loop. Consider

$$
\begin{gathered}
{ }^{k} \mathbf{x}_{b}={ }^{k} \mathbf{x}_{\text {best }} \\
{ }^{k} f_{b}=\min _{1 \leq i \leq N}\left[{ }^{k} f_{i}\left({ }^{k} \mathbf{x}_{i}\right)\right],
\end{gathered}
$$

where best $=\operatorname{argmin}_{1 \leq i \leq N}\left[{ }^{k} f_{i}\left({ }^{k} \mathbf{x}_{i}\right)\right]$ and $\operatorname{argmin}[\cdot]$ is the function to transfer a number of the individuals, which minimizes the value of the fitness function.

In the next step, new identification parameters including the tension of a cable are investigated using (7). The new search point is defined as

$$
{ }^{k+1} \overline{\mathbf{x}}_{i}=\left[1-{ }^{k} \xi_{i}\right]^{k} \mathbf{x}_{i}+{ }^{k} \xi_{i}{ }^{k} x_{b}
$$

where ${ }^{k+1} \overline{\mathbf{x}}_{i}\left(=\left[\begin{array}{llllll}k & \bar{x}_{i}^{1} & k & \bar{x}_{i}^{2} & \ldots & k \\ \bar{x}_{i}^{j} & \ldots & k & \bar{x}_{i}^{p}\end{array}\right]^{\mathrm{T}} \in \mathbb{R}^{p}\right)$ that is the $i$ th real number row vector provided newly is used to form the $(k+1)$ th mating pool and ${ }^{k} \xi_{i}\left(=\eta_{i}\left[{ }^{k} f_{b}-{ }^{k} f_{i}\right] /{ }^{k} f_{b}\right)$ denotes the coefficient of linear combination in which $\eta_{i}$ applies a positive constant between 0 and 2 .

It may be noted that the next search point ${ }^{k+1} \bar{x}_{i}$, selected sequentially by the linear combination of ${ }^{k} x_{i}$ and ${ }^{k} x_{b}$, can be the best gene to have survived from the previous iteration. Then, the identification parameters can be encoded in binary digits to form a gene for each individual as shown in the following expressions:

$$
{ }^{k+1} \overline{\mathbf{P}}=\left[\begin{array}{llllll}
{ }^{k+1} \overline{\boldsymbol{\chi}}_{1} & { }^{k+1} \overline{\boldsymbol{\chi}}_{2} & \ldots & { }^{k+1} \overline{\boldsymbol{\chi}}_{i} & \ldots & { }^{k+1} \overline{\boldsymbol{\chi}}_{N}
\end{array}\right]^{\mathrm{T}} .
$$


Once the parent genes are selected by a new h-GA reproduction process through (1) to (9), matings take place using the existing crossover scheme and then a temporary offspring matrix is produced as

$$
{ }^{k+1} \widetilde{\mathbf{P}}=\left[\begin{array}{lllll}
{ }^{k+1} & \tilde{\boldsymbol{\chi}}_{1}{ }^{k+1} \tilde{\boldsymbol{\chi}}_{2} & \ldots & { }^{k+1} \tilde{\boldsymbol{\chi}}_{i} & \ldots{ }^{k+1} \tilde{\boldsymbol{\chi}}_{N}
\end{array}\right]^{\mathrm{T}} .
$$

Then, the purpose of the final loop of the h-GA operation is to introduce diversity by mutation, in order to explore other areas in the search space. It is suggested that the mutation probability should be lowered adaptively as the process converges. Finally, through the mutation process, the following group matrix for the $(k+1)$ th generation can be obtained:

$$
{ }^{k+1} \mathbf{P}=\left[\begin{array}{lllllllll}
{ }^{k+1} \boldsymbol{\chi}_{1} & { }^{k+1} & \boldsymbol{\chi}_{2} & { }^{2} & { }^{k+1} & \boldsymbol{\chi}_{i} & { }^{k} & { }^{k+1} & \boldsymbol{\chi}_{N}
\end{array}\right]^{\mathrm{T}} .
$$

The group matrix ${ }^{k+1} \mathbf{P}$ for the $(k+1)$ th generation is used as initial information of LOA in the system updating algorithm (SUA) after performing the repeated operation up to the preassigned number for h-GA of GOA. In the case of applying methods to detect the tensile forces using LOA, the proposed h-GA provides initial values for better efficiency, especially for insufficient information at the initial stage.

In order to decide a proper bifurcation between GOA and LOA, the number of generations in h-GA and iterations in SUA should be assigned in advance. In this study, the relationship between the number of generations $\left(k_{G}\right)$ in h-GA and the number of identification parameters $(p)$ is assumed to be linear, and the number of iterations $\left(k_{L}\right)$ in SUA also can be preassigned for computational efficiency using the $k_{G}$ as

$$
\begin{gathered}
k_{G}=N_{a} p \\
k_{L}=\text { Ceil }\left[\frac{k_{G}}{2 p}\right],
\end{gathered}
$$

where $N_{a}$ denotes the preassigned arbitrary integer and Ceil[.] is the function to transfer the smallest following integer.

After iterative calculations for $k_{G}$, the final identification vector can be obtained as

$$
{ }^{k_{G}+1} \mathbf{x}_{b}=\left[\begin{array}{lllllllll}
k_{G}+1 & x_{b}^{1} & k_{G}+1 & x_{b}^{2} & \ldots & { }^{k_{G}+1} x_{b}^{j} & \ldots & { }^{k_{G}+1} x_{b}^{p}
\end{array}\right]^{\mathrm{T}},
$$

where $\left(k_{G}+1\right)$ means the first iteration step of SUA in LOA. After the termination of the h-GA procedure, the identification vector for the $r$ th $\left(1 \leq r \leq k_{L}\right)$ iteration in SUA can be assumed as

$$
{ }^{r} \mathbf{x}=\left[\begin{array}{lllllll}
{ }^{r} x^{1} & { }^{r} & x^{2} & \ldots & { }^{r} x^{j} & \ldots & { }^{r} x^{p}
\end{array}\right]^{\mathrm{T}} .
$$

Then, the static displacement and tensile force distribution can be produced for the identification vector, which is similar to the h-GA process. In the next step, the natural frequencies are determined from the finite element vibration model using the static displacement curve and tensile force distribution. Using the change in natural frequencies for different identification parameters, the sensitivity matrix $\left({ }^{r} \mathbf{S}\right)$ with $(q \times p)$ size can be determined approximately as follows:

$$
{ }^{r} \mathbf{S}=\left[\begin{array}{cccccc}
\frac{\partial^{r} \omega_{1}}{\partial^{r} x^{1}} \frac{\partial^{r} x^{1}}{\partial^{r} \omega_{1}} & \frac{\partial^{r} \omega_{1}}{\partial^{r} x^{2}} \frac{\partial^{r} x^{2}}{\partial^{r} \omega_{1}} & \cdots & \frac{\partial^{r} \omega_{1}}{\partial^{r} x^{j}} \frac{\partial^{r} x^{j}}{\partial^{r} \omega_{1}} & \cdots & \frac{\partial^{r} \omega_{1}}{\partial^{r} x^{p}} \frac{\partial^{r} x^{p}}{\partial^{r} \omega_{1}} \\
\frac{\partial^{r} \omega_{2}}{\partial^{r} x^{1}} \frac{\partial^{r} x^{1}}{\partial^{r} \omega_{2}} & \frac{\partial^{r} \omega_{2}}{\partial^{r} x^{2}} \frac{\partial^{r} x^{2}}{\partial^{r} \omega_{2}} & \cdots & \frac{\partial^{r} \omega_{2}}{\partial^{r} x^{j}} \frac{\partial^{r} x^{j}}{\partial^{r} \omega_{2}} & \cdots & \frac{\partial^{r} \omega_{2}}{\partial^{r} x^{p}} \frac{\partial^{r} x^{p}}{\partial^{r} \omega_{2}} \\
\vdots & \vdots & \vdots & \vdots & \vdots \\
\frac{\partial^{r} \omega_{i}}{\partial^{r} x^{1}} \frac{\partial^{r} x^{1}}{\partial^{r} \omega_{i}} & \frac{\partial^{r} \omega_{i}}{\partial^{r} x^{2}} \frac{\partial^{r} x^{2}}{\partial^{r} \omega_{i}} & \cdots & \frac{\partial^{r} \omega_{i}}{\partial^{r} x^{j}} \frac{\partial^{r} x^{j}}{\partial^{r} \omega_{i}} & \cdots & \frac{\partial^{r} \omega_{i}}{\partial^{r} x^{p}} \frac{\partial^{r} x^{p}}{\partial^{r} \omega_{i}} \\
\vdots & \vdots & & \vdots & \\
\frac{\partial^{r} \omega_{q} \frac{\partial^{r} x^{1}}{\partial^{r} x^{1}} \frac{\partial^{r} \omega_{q}}{\partial^{r} \omega_{q}}}{\frac{\partial^{r} x^{2}}{\partial^{r} \omega^{2}}} & \cdots & \frac{\partial^{r} \omega_{q}}{\partial^{r} x^{j}} \frac{\partial^{r} x^{j}}{\partial^{r} \omega_{q}} & \cdots & \frac{\partial^{r} \omega_{q}}{\partial^{r} x^{p}} \frac{\partial^{r} x^{p}}{\partial^{r} \omega_{q}}
\end{array}\right],
$$

where ${ }^{r} x^{j}(j=1, \ldots, p)$ and ${ }^{r} \omega_{i}(i=1, \ldots, q)$ denote the $j$ th component of the identification vector and the $i$ th mode natural frequency for the $r$ th iteration in SUA, respectively.

Then, from the produced natural frequency data, the rate of change $\left(d^{r} \boldsymbol{\omega}\right)$ for the eigenvalue can be obtained as

$$
\begin{aligned}
& d^{r} \boldsymbol{\omega} \\
& =\left[\begin{array}{llllll}
\frac{\omega_{1}^{*}-{ }^{r} \omega_{1}}{{ }^{r} \omega_{1}} & \frac{\omega_{2}^{*}-{ }^{r} \omega_{2}}{{ }^{r} \omega_{2}} & \cdots & \frac{\omega_{i}^{*}-{ }^{r} \omega_{i}}{{ }^{r} \omega_{i}} & \cdots & \frac{\omega_{q}^{*}-{ }^{r} \omega_{q}}{{ }^{r} \omega_{q}}
\end{array}\right]^{\mathrm{T}},
\end{aligned}
$$

where $\omega_{i}^{*}$ and ${ }^{r} \omega_{i}$ denote natural frequencies measured from experiment or field test and produced from nonlinear finite element vibration analysis using ${ }^{r} \mathbf{x}$ in the $r$ th iteration, respectively. Equation (16) can be rewritten in vector form using (15) as

$$
d^{r} \boldsymbol{\omega}_{(q \times 1)}={ }^{r} \mathbf{S}_{(q \times p)} d^{r} \mathbf{x}_{(p \times 1)} .
$$


Equation (17) is referred to as a linear sensitivity equation, and the rate of change for the identification vector by using (17) can be expressed as

$$
d^{r} \mathbf{x}={ }^{r} \mathbf{S}^{-1} d^{r} \boldsymbol{\omega}
$$

where ${ }^{r} \mathbf{S}^{-1}$ means the pseudoinverse matrix for ${ }^{r} \mathbf{S}$ and can be determined as

$$
{ }^{r} \mathbf{S}^{-1}=\left({ }^{r} \mathbf{S}^{\mathrm{T} r} \mathbf{S}\right)^{-1}{ }^{r} \mathbf{S}^{\mathrm{T}}
$$

Finally, the $j$ th identification parameter in the $(r+1)$ th iteration can recalculated as

$$
\begin{gathered}
{ }^{r+1} x^{j}=\left(1+d^{r} x^{j}\right)^{r} x^{j} \\
{ }^{r+1} \mathbf{x}=\left[\begin{array}{llllll}
{ }^{r+1} x^{1} & { }^{r+1} x^{2} & \ldots & { }^{r+1} x^{j} & \ldots & \\
& r+1 & x^{p}
\end{array}\right]^{\mathrm{T}} .
\end{gathered}
$$

From (13) to (20), the loop is repeated until the termination condition is satisfied. In the case of not preassigning the number of iterations $\left(k_{L}\right)$ in SUA, the convergence condition for termination of SUA process can be determined by the predefined object function that is the square roots of the sum of square (SRSS) in the following:

$$
\begin{aligned}
& \mid \sqrt{\left(\sum_{i=1}^{q}\left|\frac{\omega_{i}^{*}-{ }^{r} \omega_{i}}{\omega_{i}^{*}}\right| \times 100\right)^{2}} \\
& \quad-\sqrt{\left(\sum_{i=1}^{q}\left|\frac{\omega_{i}^{*}-{ }^{r+1} \omega_{i}}{\omega_{i}^{*}}\right| \times 100\right)^{2}} \mid<0.01 .
\end{aligned}
$$

Finally, the tension of a cable is determined from the identification parameters holding at the termination stage and relevant natural frequencies can also be determined through the finite element vibration analysis using the identification parameters.

\section{Benchmark Numerical Tests}

Before the field application of the tension estimation method using the h-SI method, the benchmark numerical tests are carried out to verify the development theory. In addition, the results of the estimations of the tension force using the characteristic equation of the mathematical model, the system identification method based on the LOA and GOA, and the proposed h-SI method are compared to investigate the accuracy and applicability of the proposed h-SI method.

Figure 2 shows a sagged cable model for benchmark numerical tests, and the mechanical and material properties of four types $(\mathrm{B} 1 \sim \mathrm{B} 4)$ of cables used in this study are summarized in Table 1 . In the table, $\lambda^{2}$ and $\xi$ represent characteristics of cables that mean dimensionless sag-extensibility and bending-stiffness parameters, respectively. When we consider the calculated values shown in Table 1, B1 is a slope cable with a very small sag and high bending stiffness and B2 is a slope cable with large sag and a moderate bending stiffness. Also, B3 means a slope cable with moderate sag and

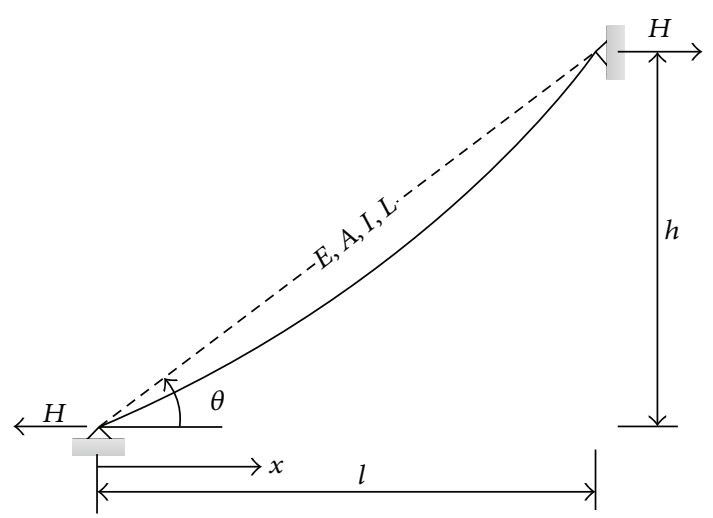

FIGURE 2: The sagged cable mode for benchmark numerical tests.

an intermediate bending stiffness, while B4 means a slope cable with intermediate sag and high bending stiffness.

The finite element models of the cables used in the benchmark numerical tests were modeled with 60 linear beam elements of the same length. Also, in the repetitive calculation phase of the h-SI method, the finite element model used for vibration analysis is renewed at the time when the system identification parameters are updated. The process of renewal can be summarized as follows. First, for the identification parameters that are updated at each repetition, a geometric nonlinear finite element analysis under the weight of the cable is performed to calculate the static sag and distribution of the tension force. Then, a finite element model that has a static sag curve and a calculated distribution of tension force is constructed. After that, the vibration analysis is performed to calculate the natural frequency.

To estimate the tension force of benchmark numerical examples for a slope cable including sags using the h-SI method, the first thing that should be done is to select the system identification parameters. In this benchmark numerical study, the tension of cables is characterized by three independent identification parameters of horizontal force $(H)$, effective diameter $\left(d_{e}\right)$, and effective mass per unit length $\left(m_{e}\right)$ of the cable. Here, the reason why the effective nominal diameter and the mass per unit length in addition to the horizontal force of the cable are included as the identification parameters is that a real cable is not of a full-section, and therefore it is necessary to calculate the precise nominal diameter to estimate the accurate tension of cables. For the tension force estimation method using the existing mathematical characteristic, errors were created as they assumed the cables to be of a full-section. Table 2 shows the search range of the identification parameters used in $h$ GA, which is a GOA of the h-SI method.

Since we set the value of $N_{a}$ at 25 in this numerical study, the maximum number $\left(k_{G}\right)$ of generations of GOA that applies h-GA is 75 , while the maximum number $\left(k_{L}\right)$ of calculations of LOA that applies SUA is 38, making a total of 113 repetitions. Table 3 shows the results of the 113 repetitive calculations using the h-SI method. Except for the B1 cable with a very small sag and high bending stiffness, all cases show an error of $1 \%$ or less. This is evidence that they were 
TABLE 1: Material and geometrical properties for four numerical inclined cable models.

\begin{tabular}{lcccc}
\hline \multirow{2}{*}{ Items } & \multicolumn{4}{c}{ Material and geometrical properties } \\
& $\mathrm{B} 1$ & $\mathrm{~B} 2$ & $\mathrm{~B} 3$ & $\mathrm{~B} 4$ \\
\hline$\lambda^{2}$ & 0.079 & 5075.8 & 1.41 & 0.508 \\
$\xi$ & 1923.5 & 3.0295 & 50.459 & 505.113 \\
$\theta\left({ }^{\circ}\right)$ & 30 & 30 & 30 & 30 \\
$L(\mathrm{~m})$ & 100 & 100 & 100 & 100 \\
$m(\mathrm{~kg} / \mathrm{m})$ & 400 & 400 & 400 & 400 \\
$H(\mathrm{MN})$ & 2.9036 & 0.7259 & 26.1325 & 0.7259 \\
$E(\mathrm{GPa})$ & 1.5988 & 17.186 & 20826.0 & 0.00478 \\
$A\left(10^{-3} \mathrm{~m}^{2}\right)$ & 7.8507 & 7.6110 & 7.8633 & 273.45 \\
$d(\mathrm{~m})$ & 0.1 & 0.984 & 0.1001 & 0.5901 \\
$I\left(10^{-6} \mathrm{~m}^{4}\right)$ & 4.9535 & 4.6097 & 4.9204 & 5950.6 \\
\hline
\end{tabular}

TABLE 2: The search range of identification variable.

\begin{tabular}{lc}
\hline Parameters & Search range \\
\hline$H(\mathrm{MN})$ & {$\left[\begin{array}{cc}0 & 30\end{array}\right]$} \\
$d_{e}(\mathrm{~m})$ & {$\left[\begin{array}{cc}10^{-3} & 1.0\end{array}\right]$} \\
$m_{e}(\mathrm{~kg} / \mathrm{m})$ & {$\left[\begin{array}{cc}10^{-3} & 500\end{array}\right]$} \\
\hline
\end{tabular}

TABLE 3: Estimated identification parameters (total number of iterative computation: 113).

\begin{tabular}{lcccc}
\hline \multirow{2}{*}{ Parameters } & \multicolumn{4}{c}{ Estimated results } \\
& $\mathrm{B} 1$ & $\mathrm{~B} 2$ & $\mathrm{~B} 3$ & $\mathrm{~B} 4$ \\
\hline$H(\mathrm{MN})$ & 2.749 & 0.726 & 26.058 & 0.730 \\
& $(-5.324)$ & $(-0.041)$ & $(-0.285)$ & $(0.620)$ \\
$d_{e}(\mathrm{~m})$ & 0.097 & 0.984 & 0.100 & 0.592 \\
& $(-3.000)$ & $(-0.010)$ & $(-0.100)$ & $(0.305)$ \\
$m_{e}(\mathrm{~kg} / \mathrm{m})$ & 378.735 & 399.800 & 398.856 & 402.530 \\
& $(-5.316)$ & $(-0.050)$ & $(-0.286)$ & $(0.632)$ \\
\hline Elapsed time $(\mathrm{sec})$ & 0.65 & 0.56 & 0.57 & 0.58 \\
\hline
\end{tabular}

(): identification error (\%).

capable of precisely estimating the tension force. For B1, it was not possible to guarantee the convergence of the final value with 113 repetitive calculations. Therefore, it was necessary to increase the number of calculations of the SUA of LOA so after more than 300 repetitive calculations, the $H$ value was calculated as $2.9035 \mathrm{MN}$, which reduced the errors to approximately $0.001 \%$.

Figure 3 shows the convergence tendency of the lateral force of B1 B4 cables. The cases of B2 4, other than B1, converge to the final value after 113 repetitive calculations. The B1 case shows a change in the value of the lateral force during being identified after the 98th repetitive calculation, hinting that it is following a track of continuous convergence process. Therefore, in the case of B1, the number of LOA's repetitive calculations should be increased to reduce identification errors. As shown in the figure, the GOA, which is operated to provide initial value information for LOA, converges at relatively early generations. It needs to be noticed that the GOA's h-GA provides information on the initial value of the LOA’s SUA very effectively.

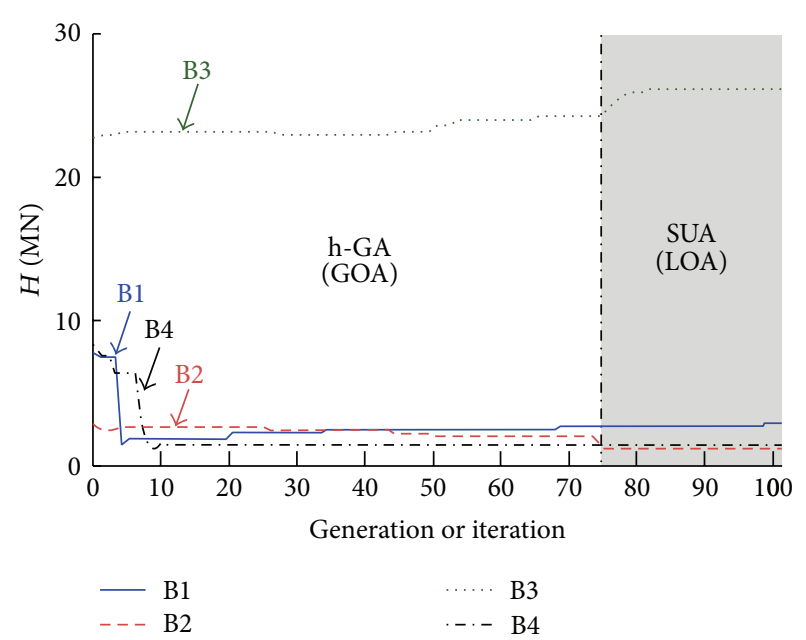

FIGURE 3: The convergence processes to find the horizontal force for B1 4.

The proposed method using the h-SI is compared with those of various existing approaches as listed in Table 4. The table reveals that these works, based on analytical approaches, have limited capabilities in dealing with the tension detection of cables, primarily due to their limitations in handling different geometries of cables and bending stiffness effects in the analysis. It should be also noted that the approach using the characteristic equation of beams could result in model error, especially for cables with large sag.

In the case of applying SUA and h-SI, it shows excellent detectability as errors were within $0.63 \%$, except for B1, because it uses the FE model which reflects the geometrical characteristics of cables such as the sag. For the same iteration (113 times), the detectability for the case of B1 is lower than others because of their complexity due to small sagged geometry and large flexural stiffness. It means that, for B1, it is not possible to guarantee the convergence of the final value with 113 repetitive calculations. Therefore, it is necessary to increase the number of calculations of SUA of LOA in order to obtain an optimal solution.

Table 5 shows the results of estimations of the identification parameters using the SUA methods with and $90 \%$ 
TABLE 4: Estimation results of cable tension using various existing approaches.

\begin{tabular}{|c|c|c|c|c|}
\hline \multirow{2}{*}{ Methods } & \multicolumn{4}{|c|}{ Estimated cable tension $(\mathrm{MN})$} \\
\hline & B1 & B2 & B3 & B4 \\
\hline Taut string theory & $\begin{array}{c}3.325 \\
(-0.048)\end{array}$ & $\begin{array}{c}112.346 \\
(>100)\end{array}$ & $\begin{array}{c}44.789 \\
(48.430)\end{array}$ & $\begin{array}{c}0.838 \\
(-0.522)\end{array}$ \\
\hline Triantafyllou and Grinfogel [16] & $\begin{array}{c}3.352 \\
(-0.057)\end{array}$ & $\begin{array}{c}0.815 \\
(-3.287)\end{array}$ & $\begin{array}{c}31.330 \\
(3.828)\end{array}$ & $\begin{array}{c}0.841 \\
(-0.202)\end{array}$ \\
\hline Linear regression method & $\begin{array}{c}3.352 \\
(-0.069)\end{array}$ & $\begin{array}{c}0.690 \\
(-18.073)\end{array}$ & $\begin{array}{c}30.160 \\
(-0.049)\end{array}$ & $\begin{array}{c}0.835 \\
(-0.973)\end{array}$ \\
\hline Zui et al. [1] & $\begin{array}{c}3.354 \\
(-0.012)\end{array}$ & $\begin{array}{c}2.940 \\
(>100)\end{array}$ & $\begin{array}{c}29.031 \\
(-3.791)\end{array}$ & $\begin{array}{c}0.685 \\
(-18.696)\end{array}$ \\
\hline Sensitivity updating algorithm & $\begin{array}{c}3.106 \\
(-7.391)\end{array}$ & $\begin{array}{c}0.838 \\
(-0.558)\end{array}$ & $\begin{array}{c}30.089 \\
(-0.285)\end{array}$ & $\begin{array}{c}0.848 \\
(0.629)\end{array}$ \\
\hline Microgenetic algorithm ( $\mu-\mathrm{GA})$ & $\begin{array}{c}3.362 \\
(0.242)\end{array}$ & $\begin{array}{c}1.398 \\
(65.895)\end{array}$ & $\begin{array}{c}27.736 \\
(-8.083)\end{array}$ & $\begin{array}{c}0.723 \\
(-14.204)\end{array}$ \\
\hline This study (h-GA + SUA) & $\begin{array}{c}3.174 \\
(-5.634)\end{array}$ & $\begin{array}{c}0.838 \\
(-0.558)\end{array}$ & $\begin{array}{c}30.089 \\
(-0.285)\end{array}$ & $\begin{array}{c}0.843 \\
(0.036)\end{array}$ \\
\hline True cable tension $\left(T_{\text {true }}\right)$ & 3.354 & 0.843 & 30.175 & 0.843 \\
\hline
\end{tabular}

( ): identification error (\%).

TABLE 5: Influence of initial information of identification parameters on the estimated results.

\begin{tabular}{|c|c|c|c|c|}
\hline \multirow{2}{*}{ Case } & \multirow{2}{*}{ Initial value } & \multicolumn{3}{|c|}{ Identification parameters } \\
\hline & & $H(\mathrm{MN})$ & $d(\mathrm{~m})$ & $m(\mathrm{~kg} / \mathrm{m})$ \\
\hline \multirow{3}{*}{ B1 } & ${ }^{0} x^{j}(\operatorname{Max})$ & 5.547 & 0.139 & 764.340 \\
\hline & ${ }^{0} x^{j}$ (90\% of True) & 2.690 & 0.096 & 370.587 \\
\hline & ${ }^{0} x^{j}(\operatorname{Min})$ & $*$ & $*$ & $*$ \\
\hline \multirow{3}{*}{ B2 } & ${ }^{0} x^{j}(\operatorname{Max})$ & * & * & * \\
\hline & ${ }^{0} x^{j}(90 \%$ of True $)$ & 0.726 & 0.984 & 399.800 \\
\hline & ${ }^{0} x^{j}(\operatorname{Min})$ & $*$ & $*$ & $*$ \\
\hline \multirow{3}{*}{ B3 } & ${ }^{0} x^{j}(\operatorname{Max})$ & $*$ & $*$ & $*$ \\
\hline & ${ }^{0} x^{j}(90 \%$ of True $)$ & 26.058 & 0.100 & 398.856 \\
\hline & ${ }^{0} x^{j}(\operatorname{Min})$ & * & $*$ & $*$ \\
\hline \multirow{3}{*}{ B4 } & ${ }^{0} x^{j}(\operatorname{Max})$ & 5.126 & 0.997 & 2828.500 \\
\hline & ${ }^{0} x^{j}(90 \%$ of True $)$ & 0.734 & 0.592 & 402.530 \\
\hline & ${ }^{0} x^{j}(\operatorname{Min})$ & $*$ & $*$ & $*$ \\
\hline
\end{tabular}

$*$ : numerical divergence or local minimum.

of actual values of Table 1 and maximum and minimum values of Table 2. All numerical tests confirm the possibility that the wrong selection of initial information may result in numerical divergence or local minimum. This emphasizes the significance of the information at the initial stage when applying SUA, which is one of the drawbacks of the LOA method. If the initial value is set similar to the actual value, the estimated errors can be reduced and the calculation time for LOA can be reduced. However, in actual field, the information on the initial values is very limited, and a repetitive process of trial and error is inevitable.

Since the $\mu$-GA is dealing with small populations, it is unlikely that the process converges into the true value in the first iteration loop. In this case, we need more iteration to converge into the accurate value. Note that the tension force detection of cables in this case is heavily dependent on the number of iterations and initial information. On the other hand, the h-SI method using a combination of GOA and LOA shows the excellent detectability for all cases. This shows that the tension estimation technique using the proposed h-SI can provide good initial information for SUA. Table 6 shows elapsed times of each system identification method after 113 iterative generations or computing. We may conclude from the table that the computational performance of the h-SI is equal to or greater improvement than the $\mu$-GA and SUA while maintaining the same level of accuracy.

\section{Field Applicability Tests}

To investigate field applicability of the proposed h-SI method, we considered the time series data measured by the four accelerometers that were installed by 2 on each side of an actual cable-stayed bridge (cables number 1 and number 44, Seohae Bridge). As shown in Figure 4, the cross-section 
TABLE 6: Comparison of elapsed time of each system identification method after 113 iterative generations or computing.

\begin{tabular}{|c|c|c|c|c|}
\hline \multirow{2}{*}{ System identification methods } & \multicolumn{4}{|c|}{ Elapsed time (sec) } \\
\hline & B1 & $\mathrm{B} 2$ & B3 & B4 \\
\hline SUA $\left[{ }^{0} x^{j}(\operatorname{Max})\right]$ & 0.62 & $*$ & $*$ & 1.48 \\
\hline SUA $\left[{ }^{0} x^{j}\right.$ (90\% of True) $]$ & 1.12 & 1.29 & 1.18 & 1.31 \\
\hline SUA $\left[{ }^{0} x^{j}(\operatorname{Min})\right]$ & $*$ & $*$ & $*$ & $*$ \\
\hline$\mu$-GA & 0.72 & 0.69 & 0.61 & 0.72 \\
\hline h-SI (This study) & 0.65 & 0.56 & 0.57 & 0.58 \\
\hline
\end{tabular}

*: numerical divergence or local minimum.

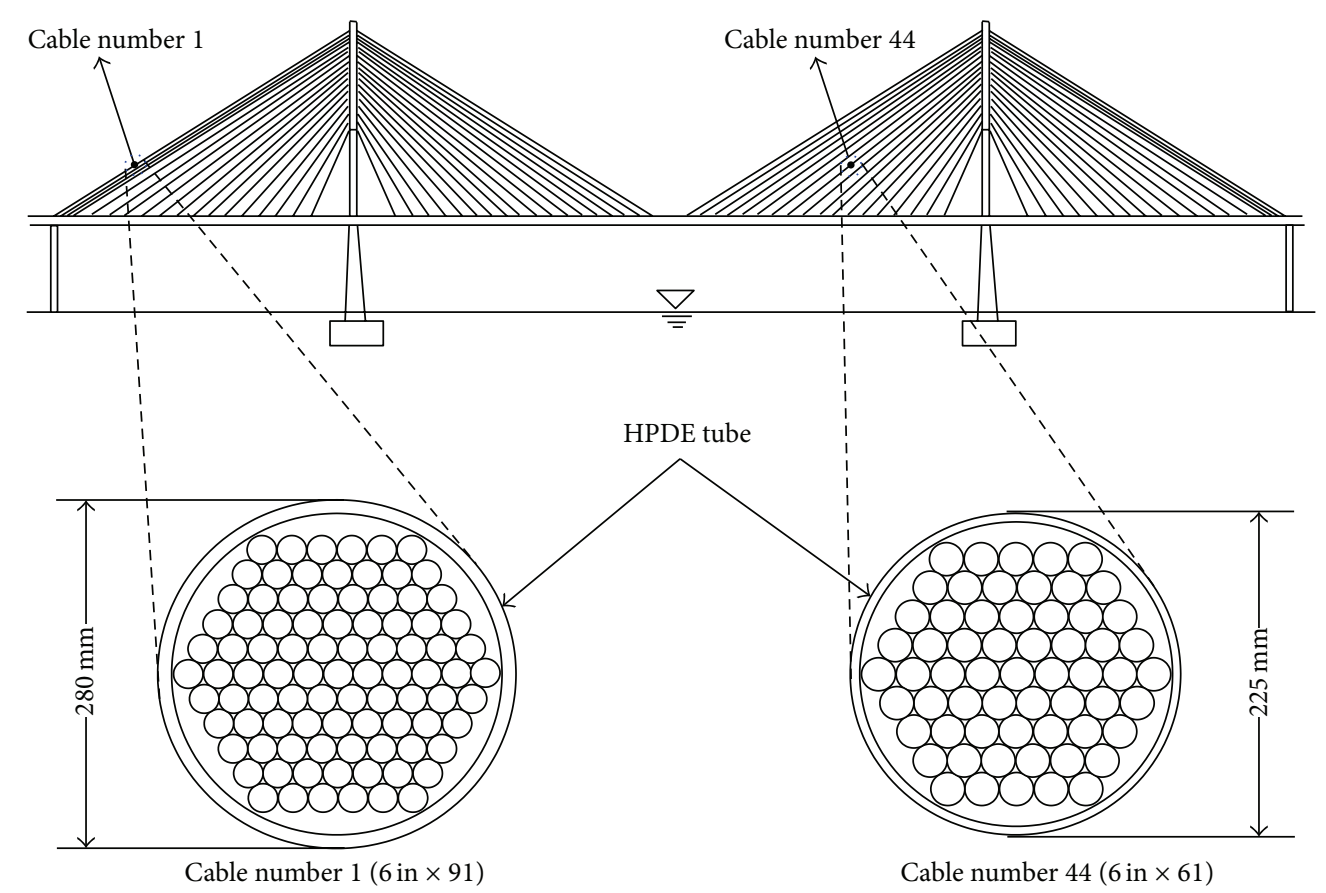

Figure 4: The locations, number, and section of measured cables.

of cable number 1 is composed of 91 strands of which the nominal diameter $(\phi)$ is $0.0157 \mathrm{~m}(0.6 \mathrm{in})$. The cable is shielded in HPDE pipes. Cable number 1 is the longest and vulnerable to vibration. The weight of cable number 1 is $125 \mathrm{kgf} / \mathrm{m}$, which is also the heaviest. The cross-section of cable number 44 is composed of 61 strand wires with the same diameter as those in cable number 1 . The cable is also shielded by HPDE tubes. The weight of cable number 44 is $84 \mathrm{kgf} / \mathrm{m}$. Here, to build a necessary finite element model for the system identification, an ideal sloped cable as shown in Figure 5 is constructed.

Using a Piezoelectric type accelerometer, time series acceleration data perpendicular to the longitudinal direction of the cables on both sides were obtained. Here, the sampling frequency was $0.01 \mathrm{sec}$, while the average wind velocity during the measurement was $4.23 \mathrm{~m} / \mathrm{s}$. The ambient temperature was $-1.76^{\circ} \mathrm{C}$. The time series data of the acceleration measurement was examined using Welch's method $(\mathrm{NFFT}=2048$, Hanning window) to identify the acceleration spectrum. Also, by using the ERADC [13] method, the natural frequency was also extracted. Tables 7 and 8 show the natural frequencies extracted by the finite element model with 100 elements and the natural frequencies measured from cables number 1 and number 44 on each side of the bridge. The material and geometrical properties of the cables for the finite element analysis are shown in Table 9.

The result of cable tension force estimation using the existing methods and the proposed h-SI method based on the natural frequency obtained through the field measurement is shown in Table 10. In addition, using the SUA, $\mu-G A$, and h-SI methods, the results of estimating the lateral forces and effective nominal diameters, which are two identification parameters, are shown in Table 11.

The estimation of the tension force using the taut string theory yields average tension forces of $5.586 \mathrm{MN}$ and $5.532 \mathrm{MN}$ for cable number 1 on each side of the bridge, while cable number 44 yields $5.226 \mathrm{MN}$ and $5.253 \mathrm{MN}$, respectively. Here, the effective lengths of the cable applied were $228.86 \mathrm{~m}$ and $165.33 \mathrm{~m}$, while the mass per unit length was as per the reported actual physical properties of the actual cables. The method of Triantafyllou and Gingfogel, which estimates the average tension force from nonlinear 
TABLE 7: Extracted natural frequencies from measured data and calculated natural frequencies from FE models for cable number 1 (Hz).

\begin{tabular}{|c|c|c|c|c|c|c|}
\hline \multirow{2}{*}{ Mode number } & \multicolumn{3}{|c|}{ Upward-bound } & \multicolumn{3}{|c|}{ Downward-bound } \\
\hline & Test & FEM & Error $(\%)$ & Test & FEM & Error (\%) \\
\hline 1 & 0.47 & 0.46 & -1.71 & - & 0.46 & - \\
\hline 2 & 0.94 & 0.92 & -1.87 & 0.92 & 0.92 & 0.48 \\
\hline 3 & - & 1.14 & - & - & 0.94 & - \\
\hline 4 & 1.38 & 1.38 & -0.28 & 1.38 & 1.38 & 0.25 \\
\hline 5 & 1.84 & 1.85 & 0.42 & 1.84 & 1.84 & 0.25 \\
\hline 6 & - & 2.28 & - & - & 1.87 & - \\
\hline 7 & 2.30 & 2.31 & 0.30 & 2.29 & 2.29 & -0.20 \\
\hline 8 & 2.76 & 2.77 & 0.19 & 2.75 & 2.75 & -0.11 \\
\hline 9 & 3.23 & 3.23 & 0.06 & - & 2.80 & - \\
\hline 10 & - & 3.42 & - & 3.21 & 3.21 & 0.06 \\
\hline 11 & 3.69 & 3.69 & -0.01 & 3.67 & 3.67 & -0.07 \\
\hline 12 & 4.15 & 4.16 & 0.24 & - & 3.74 & - \\
\hline 13 & - & 4.56 & - & 4.13 & 4.13 & 0.04 \\
\hline 14 & 4.61 & 4.62 & 0.25 & 4.59 & 4.59 & 0.07 \\
\hline 15 & 5.08 & 5.08 & 0.09 & - & 4.68 & - \\
\hline 16 & 5.54 & 5.54 & 0.03 & 5.05 & 5.05 & 0.07 \\
\hline 17 & - & 5.70 & - & 5.51 & 5.51 & -0.02 \\
\hline 18 & 6.00 & 6.00 & 0.08 & - & 5.61 & - \\
\hline 19 & 6.46 & 6.46 & -0.01 & 5.98 & 5.97 & -0.15 \\
\hline 20 & - & 6.84 & - & 6.44 & 6.43 & -0.10 \\
\hline
\end{tabular}

-: not extracted.

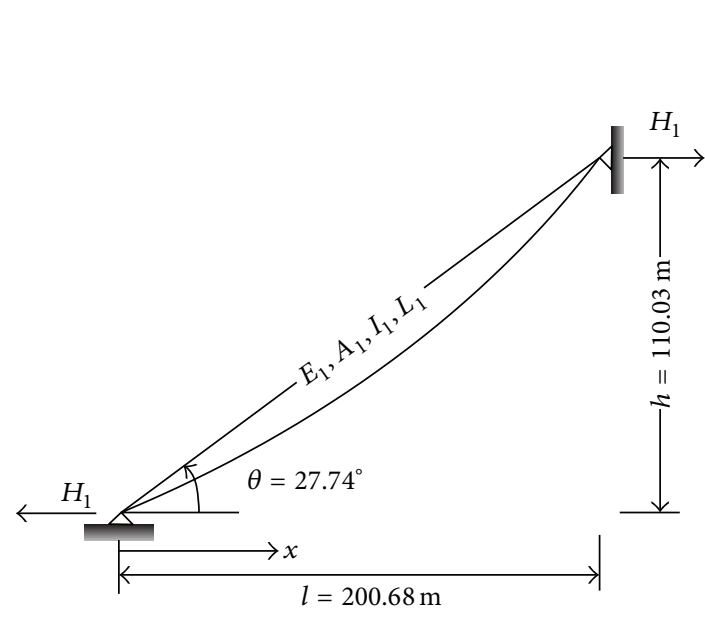

(a) Cable number 1

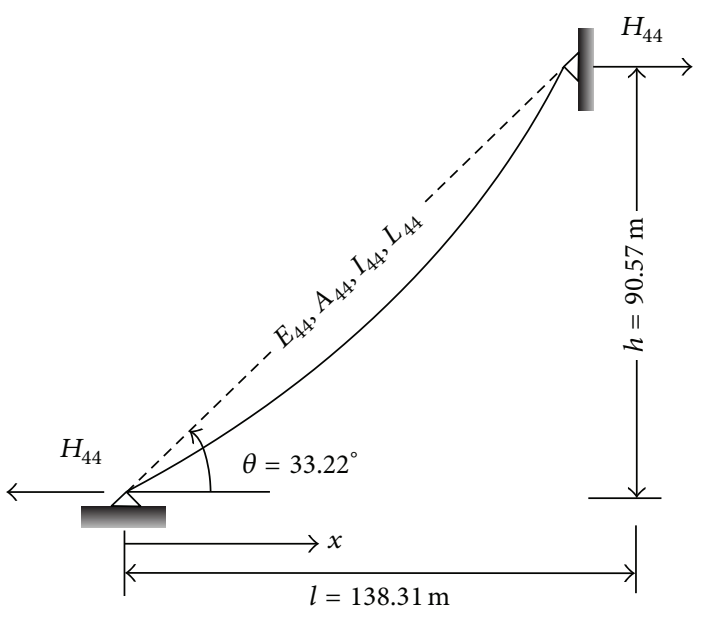

(b) Cable number 44

Figure 5: Idealized cable for FE model.

characteristics equations of the sloped cables and takes sags into consideration, shows errors within $1.4 \%$ in all cases, indicating a moderate level of precision in estimation. The mass per unit length of the cable and data on the axial stiffness of the cable were assumed to be known in advance. The estimated tension force using the linear regression method, which considers bending, ignores the sag, and uses the relationships between the natural frequency, bending stiffness, and average tension forces, shows errors within $1.8 \%$ for a relatively higher level of precision. However, when the bending stiffness was estimated, F4 case showed a negative value of bending stiffness, which was $-2.545 e+05 \mathrm{MN}$, rendering the results physically irrelevant. This shows the limitations in estimating the tension force using the linear regression method. The estimation of the tension force using the high mode method suggested by Shimada [2] shows an error range within $2.3 \%$. This is the least precise of all estimation methods. Still, the error is relatively small.

The estimation results by the SUA, $\mu$-GA, and h-SI methods, which are system identification methods, show 
TABLE 8: Extracted natural frequencies from measured data and calculated natural frequencies from FE models for cable number 44 (Hz).

\begin{tabular}{|c|c|c|c|c|c|c|}
\hline \multirow{2}{*}{ Mode number } & \multicolumn{3}{|c|}{ Upward-bound } & \multicolumn{3}{|c|}{ Downward-bound } \\
\hline & Test & FEM & Error $(\%)$ & Test & FEM & Error (\%) \\
\hline 1 & - & 0.75 & - & - & 0.76 & - \\
\hline 2 & - & 1.33 & - & - & 1.33 & - \\
\hline 3 & 1.50 & 1.51 & 0.50 & 1.51 & 1.51 & 0.33 \\
\hline 4 & 2.26 & 2.26 & 0.06 & 2.27 & 2.27 & 0.05 \\
\hline 5 & - & 2.66 & - & - & 2.67 & - \\
\hline 6 & 3.01 & 3.02 & 0.24 & 3.02 & 3.02 & 0.00 \\
\hline 7 & 3.77 & 3.77 & -0.03 & 3.78 & 3.78 & -0.12 \\
\hline 8 & - & 3.99 & - & - & 4.00 & - \\
\hline 9 & 4.52 & 4.52 & -0.02 & 4.55 & 4.54 & -0.13 \\
\hline 10 & 5.29 & 5.28 & -0.18 & 5.31 & 5.29 & -0.38 \\
\hline 11 & - & 5.32 & - & - & 5.34 & - \\
\hline 12 & 6.03 & 6.03 & -0.05 & 6.05 & 6.05 & 0.02 \\
\hline 13 & - & 6.65 & - & - & 6.67 & - \\
\hline 14 & 6.79 & 6.79 & -0.03 & 6.82 & 6.81 & -0.21 \\
\hline 15 & 7.55 & 7.54 & -0.19 & 7.58 & 7.56 & -0.27 \\
\hline 16 & - & 7.99 & - & - & 8.01 & - \\
\hline 17 & 8.31 & 8.3 & -0.09 & 8.33 & 8.32 & -0.17 \\
\hline 18 & 9.06 & 9.05 & -0.09 & 9.09 & 9.07 & -0.25 \\
\hline 19 & - & 9.32 & - & - & 9.35 & - \\
\hline 20 & 9.81 & 9.81 & -0.03 & 9.83 & 9.83 & -0.02 \\
\hline
\end{tabular}

-: not extracted.

TABLE 9: The material and geometrical properties of each cable.

\begin{tabular}{|c|c|c|c|c|}
\hline \multirow{2}{*}{ Condition } & \multicolumn{4}{|c|}{ Tests } \\
\hline & $\mathrm{F} 1$ & F2 & F3 & $\mathrm{F} 4$ \\
\hline$\lambda^{2}$ & 0.0147 & 0.0093 & 0.0021 & 0.0017 \\
\hline$\xi$ & 1455.3 & 873.1 & 800.5 & 4313.9 \\
\hline Horizontal load (MN) & 4.8940 & 4.8341 & 4.3652 & 4.3938 \\
\hline Effective diameter (m) & 0.280 & 0.280 & 0.225 & 0.225 \\
\hline Elasticity (GPa) & 2.1 & 2.1 & 2.1 & 2.1 \\
\hline Remark & Number 1 (up) & Number 1 (down) & Number 44 (up) & Number 44 (down) \\
\hline
\end{tabular}

TABLE 10: Cable tension force estimated from existing methods and the h-SI method.

\begin{tabular}{|c|c|c|c|c|}
\hline \multirow{2}{*}{ Methods } & \multicolumn{4}{|c|}{ Estimated cable tension (MN) } \\
\hline & $\mathrm{F} 1$ & $\mathrm{~F} 2$ & F3 & $\mathrm{F} 4$ \\
\hline Taut string theory & $\begin{array}{c}5.586 \\
(-1.000)\end{array}$ & $\begin{array}{c}5.532 \\
(-1.401)\end{array}$ & $\begin{array}{c}5.226 \\
(-1.547)\end{array}$ & $\begin{array}{c}5.253 \\
(1.152)\end{array}$ \\
\hline Triantafyllou and Grinfogel [16] & $\begin{array}{c}5.578 \\
(-1.147)\end{array}$ & $\begin{array}{c}5.534 \\
(-1.372)\end{array}$ & $\begin{array}{c}5.226 \\
(-1.545)\end{array}$ & $\begin{array}{l}5.253 \\
(1.152)\end{array}$ \\
\hline Linear regression method & $\begin{array}{c}5.571 \\
(-1.276)\end{array}$ & $\begin{array}{c}5.518 \\
(-1.650)\end{array}$ & $\begin{array}{c}5.214 \\
(-1.773)\end{array}$ & $\begin{array}{c}5.267 \\
(1.419)\end{array}$ \\
\hline Shimada [2] & $\begin{array}{c}5.549 \\
(-1.653)\end{array}$ & $\begin{array}{c}5.486 \\
(-2.233)\end{array}$ & $\begin{array}{c}5.196 \\
(-2.110)\end{array}$ & $\begin{array}{c}5.204 \\
(0.206)\end{array}$ \\
\hline Sensitivity updating algorithm & $\begin{array}{c}5.583 \\
(-1.058)\end{array}$ & $\begin{array}{c}5.525 \\
(-1.533)\end{array}$ & $\begin{array}{c}5.233 \\
(-1.413)\end{array}$ & $\begin{array}{c}5.275 \\
(1.579)\end{array}$ \\
\hline Microgenetic algorithm $(\mu-\mathrm{GA})$ & $\begin{array}{c}5.592 \\
(-0.899)\end{array}$ & $\begin{array}{c}5.514 \\
(-1.729)\end{array}$ & $\begin{array}{c}5.250 \\
(-1.093)\end{array}$ & $\begin{array}{c}5.287 \\
(1.810)\end{array}$ \\
\hline This study (h-GA + SUA) & $\begin{array}{c}5.583 \\
(-1.058) \\
\end{array}$ & $\begin{array}{c}5.525 \\
(-1.533) \\
\end{array}$ & $\begin{array}{c}5.233 \\
(-1.413) \\
\end{array}$ & $\begin{array}{c}5.272 \\
(1.521) \\
\end{array}$ \\
\hline Static jacking force $\left(T_{\text {true }}\right)$ & 5.643 & 5.611 & 5.308 & 5.193 \\
\hline
\end{tabular}

( ): identification error (\%). 
TABLE 11: Estimation results of identification parameters through various system identification methods.

\begin{tabular}{|c|c|c|c|c|}
\hline \multirow{2}{*}{ Case } & \multirow{2}{*}{${ }^{\text {final }} x^{j}$} & \multicolumn{3}{|c|}{ Methods } \\
\hline & & SUA (LOA) & $\mu-\mathrm{GA}(\mathrm{GOA})$ & $\mathrm{h}-\mathrm{SI}(\mathrm{GOA}+\mathrm{LOA})$ \\
\hline \multirow{6}{*}{$\mathrm{F} 1$} & $H(\mathrm{MN})$ & 4.892 & 4.887 & 4.892 \\
\hline & $d(\mathrm{~m})$ & 0.184 & 0.210 & 0.188 \\
\hline & $E I\left(\mathrm{Nm}^{2}\right)$ & $1.182 e+05$ & $2.005 e+05$ & $1.288 e+05$ \\
\hline & $E A(\mathrm{MN})$ & 55.840 & 73.736 & 58.294 \\
\hline & $\lambda^{2}$ & 0.033 & 0.043 & 0.034 \\
\hline & $\xi$ & 1,473 & 1,130 & 1,411 \\
\hline \multirow{6}{*}{$\mathrm{F} 2$} & $H(\mathrm{MN})$ & 4.841 & 4.832 & 4.841 \\
\hline & $d(\mathrm{~m})$ & 0.208 & 0.224 & 0.208 \\
\hline & $E I\left(\mathrm{Nm}^{2}\right)$ & $1.929 e+05$ & $2.595 e+05$ & $1.929 e+05$ \\
\hline & $E A(\mathrm{MN})$ & 71.357 & 82.757 & 71.357 \\
\hline & $\lambda^{2}$ & 0.043 & 0.051 & 0.043 \\
\hline & $\xi$ & 1,147 & 988 & 1,147 \\
\hline \multirow{6}{*}{ F3 } & $H(\mathrm{MN})$ & 4.362 & 4.887 & 4.892 \\
\hline & $d(\mathrm{~m})$ & 0.212 & 0.210 & 0.188 \\
\hline & $E I\left(\mathrm{Nm}^{2}\right)$ & $2.082 e+05$ & $2.005 e+05$ & $1.288 e+05$ \\
\hline & $E A(\mathrm{MN})$ & 55.840 & 73.736 & 58.294 \\
\hline & $\lambda^{2}$ & 0.014 & 0.010 & 0.014 \\
\hline & $\xi$ & 757 & 1,087 & 750 \\
\hline \multirow{6}{*}{$\mathrm{F} 4$} & $H(\mathrm{MN})$ & 4.892 & 4.887 & 4.892 \\
\hline & $d(\mathrm{~m})$ & 0.184 & 0.210 & 0.188 \\
\hline & $E I\left(\mathrm{Nm}^{2}\right)$ & $1.182 e+05$ & $2.005 e+05$ & $1.288 e+05$ \\
\hline & $E A(\mathrm{MN})$ & 55.840 & 73.736 & 58.294 \\
\hline & $\lambda^{2}$ & 0.014 & 0.013 & 0.012 \\
\hline & $\xi$ & 760 & 813 & 871 \\
\hline
\end{tabular}

errors within $1.6 \%, 1.9 \%$, and $1.6 \%$, respectively. These errors do not differ significantly from those of existing methods using the mathematical equations. The reason for this is that the cables of the cable-stayed bridges used in field study are part of systems that are less influenced by sags and bending compared to those of the benchmark numerical models. In fact, the values of the dimensionless sag-extensibility $\left(\lambda^{2}\right)$ for the numerical model are of a range of 0.079 5,076. However, as shown in Table 9, they are less than 0.015 in all field experiment cases, which signifies that the cables used in field study are less influenced by sags and bending.

When compared to the tension forces measured using a hydraulic jack before completion [14] all methods show errors by less than $2.3 \%$. Such errors can be regarded as the loss of cable tension force. When considering the fact that the bridge undergoes the tension loss by continuous changes of tension forces, with an accumulation of damage due to the environmental cyclic loadings such as thermal, traffic, and wind loads, the reduction rate in tension force mentioned above is insignificant. Unlike cables used in the benchmark numerical tests, the actual cables of cable-stayed bridges used in field study are systems that are less influenced by sags and bending. Therefore, the existing estimation methods and the proposed h-SI method do not differ significantly in terms of the estimation result. However, while the existing mathematical models and SUA, which are LOA, require accurate data in advance, it should be noted that the $\mu$-GA, which was GOA, and the h-SI method are not affected by such initial information. Also, compared to the $\mu$-GA, it should be noted that h-SI could improve the converging tendency toward the final value and the precision by being combined with SUA.

\section{Summary and Conclusions}

In this paper, the characteristics of the h-SI methods that could be applied to the estimation of the tension forces effectively are examined and the theoretical formulation process of the h-SI method is suggested. Also, to verify the efficiency of the h-SI tension force estimation method, benchmark numerical tests for various cases are conducted and the applicability of the h-SI method through field tests is verified. During the benchmark numerical and field studies, comparative studies with the existing characteristics equation methods and system identification methods are also carried out. The key findings from this study are summarized as follows.

The h-SI method is a new algorithm combined to use the advantages of both the LOA and GOA and adopts the posthybridization method where the GOA provides the initial values for the LOA. The most significant characteristic of this method is that the h-GA, which is a GOA, helps solve 
the local convergence problem due to incorrect initial value settings, by using the sensitivity based system identification method, which is a LOA, to improve the precision and convergence to the final value.

The benchmark numerical tests that consider sag and bending stiffness showed that, in the case of string theory, it was more appropriate for the estimation of tension force for a simple cable system where the influence of sag and bending stiffness is not significant. Also, the method suggested by Triantafyllou and Grinfogel, which considers sags, showed errors within $4 \%$ in all cases, proving to be a relatively precise method. However, as the method uses a trial and error method, it is time consuming to find the tension compared to the string theory and initial information on the mass per unit length and axial stiffness of the cable is required to estimate the tension force properly. For estimating the tension force using the linear regression method, in which bending is considered but sags are not, except for the cable with large sags, the tension force can be estimated precisely within $1 \%$ errors. However, when applying the characteristic equation of the beam under axial loading for cables with large sags, model errors were confirmed in the results of tension estimation. For the low mode method suggested by Zui et al. [1] and Yen et al. [15], which considers both bending and sag, the bending stiffness, axial stiffness, and sag ratio are required in advance to estimate cable tension accurately. For cables with larger sags or higher bending stiffness, the accuracy of the estimation of the tension force was confirmed to be undermined significantly. On the other hand, when applying SUA, which is a LOA, all showed an estimation error lower within $1.2 \%$ except for $\mathrm{B} 1$, signifying that the estimation method using SUA is effective. All benchmark numerical tests showed the possibility that the wrong selection of initial information may result in numerical divergence or local minimum. In the case of applying $\mu$-GA, which is a GOA, when exploring the solution space, it could not have a direction, and it resulted in slow convergence to the final value. Therefore, with 113 generations in total, it still did not converge to the final value showing the errors in all cases. Therefore, after 113 generations, it still did not converge to the final value and showed significant errors in all cases. The proposed h-SI technique that combined the h-GA and SUA was the most effective in estimation. In particular, in the h-SI method of estimating the tension force, the h-GA can play an important role in the initial operation of the SUA.

In the field test that used cables applied to an actual cable-stayed bridge, all methods showed errors smaller than $2.3 \%$. When considering the fact that the bridge undergoes the tension loss by continuous changes of tension forces, with an accumulation of damage due to the environmental cyclic loadings such as thermal, traffic, and wind loads, the reduction rate in tension force mentioned above is insignificant. Unlike cables used in the benchmark numerical tests, the actual cables of cable-stayed bridges used in field study are systems that are less influenced by sags and bending. Therefore, the existing estimation methods and the proposed h-SI method do not differ significantly in terms of the estimation result. However, while the existing mathematical models and the SUA, which is LOA, require precise data in advance, it should be noted that the $\mu$-GA, which is GOA, and the h-SI method are not affected by such information. Also, compared to the $\mu$-GA, it should be noted that h-SI could improve the converging tendency toward the final value and precision by being combined with SUA.

Conclusively, the proposed h-SI method could be effectively applied to estimate the tension force variation without initial information in actual cable bridges with inclined cables including sag.

\section{Conflict of Interests}

The authors declare that there is no conflict of interests regarding the publication of this paper.

\section{Acknowledgment}

This work was supported by a National Research Foundation of Korea (NRF) Grant funded by the Korean government (MEST) (no. 2012-0008762).

\section{References}

[1] H. Zui, T. Shinke, and Y. Namita, "Practical formulas for estimation of cable tension by vibration method " Journal of Structural Engineering, vol. 122, no. 6, pp. 651-656, 1996.

[2] T. Shimada, "Estimating method of cable tension from natural frequency of high mode," Proceedings of the Japan Society of Civil Engineers, vol. 501, no. 29, pp. 163-171, 1994.

[3] H. Tang, S. Xue, and C. Fan, "Differential evolution strategy for structural system identification," Computers and Structures, vol. 86, no. 21-22, pp. 2004-2012, 2008.

[4] T. Park, S. Moon, H. J. Joo, and B. H. Kim, "Estimating tensile force of hangers in suspension bridges using frequency based SI technique: I. theory," Journal of the Korean Society of Civil Engineers, vol. 27, no. 2A, pp. 165-172, 2007.

[5] S. B. Bati and U. Tonietti, "Experimental methods for estimating in situ tensile force intie-rods," Journal of Engineering Mechanics, vol. 127, no. 12, pp. 1275-1283, 2001.

[6] C. Blasi and S. Sorace, "Determining the axial force in metallic rods," Structural Engineering International (IABSE), vol. 4, pp. 241-246, 1994.

[7] S. Park, S. Choi, S.-T. Oh, N. Stubbs, and H.-C. Song, "Identification of the tensile force in high-tension bars using modal sensitivities," International Journal of Solids and Structures, vol. 43, no. 10, pp. 3185-3196, 2006.

[8] T. Park, H. T. Jang, M. H. Noh, and K. S. Park, "Evaluation on the lost prestressing force of an external tendon using the combination of FEM and HGA: I. theory," Journal of the Korean Institute for Structural Maintenance Inspection, vol. 13, no. 5, pp. 109-120, 2009.

[9] S.-Y. Lee and S.-C. Wooh, "Waveform-based identification of structural damage using the combined finite element method and microgenetic algorithms," Journal of Structural Engineering, vol. 131, no. 9, pp. 1464-1472, 2005.

[10] M. H. Noh, H. T. Jang, S. Y. Lee, and T. Park, "Tensile force estimation of externally prestressed tendon using SI technique based on differential evolutionary algorithm," Journal of the Korean Society of Civil Engineers, vol. 29, no. 1, pp. 9-18, 2009. 
[11] M. H. Noh and S. Y. Lee, "Moving load identification in time domain using a coupled genetic algorithm," in Green and Smart Technology with Sensor Applications, vol. 338 of Communications in Computer and Information Science, pp. 136143, Springer, Berlin, Germany, 2012.

[12] M.-H. Noh and J. W. Hu, "Tensile force identification in cablestayed structures: hybrid system identification algorithm and experimental verification," Journal of Mechanical Science and Technology, vol. 28, no. 11, pp. 4563-4572, 2014.

[13] J.-N. Juang, Applied System Identification, Prentice-Hall, Englewood Cliffs, NJ, USA, 1974.

[14] J. C. Park, C. M. Park, and P. Y. Song, "Evaluation of structural behaviors using full scale measurements on the Seo Hae cablestayed bridge," KSCE Journal of Civil Engineering, vol. 24, no. 2A, pp. 249-257, 2004.

[15] W. H. Yen, A. B. Mehrabi, and H. Tabatabai, "Evaluation of stay cable tension using a non-destructive vibration technique," in Building to Last Structures Congress: Proceedings of the 15th Structures Congress, L. Kempner Jr. and C. B. Brown, Eds., vol. 1, pp. 503-507, ASCE, New York, NY, USA, 1997.

[16] M. S. Triantafyllou and L. Grinfogel, "Natural frequencies and modes of inclined cables," Journal of Structural Engineering, vol. 112, no. 1, pp. 139-148, 1986. 


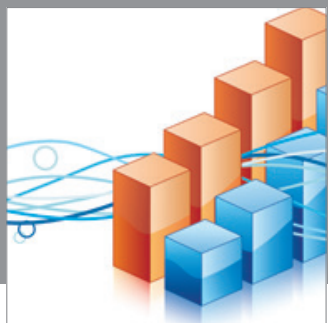

Advances in

Operations Research

mansans

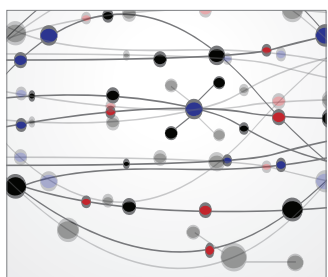

The Scientific World Journal
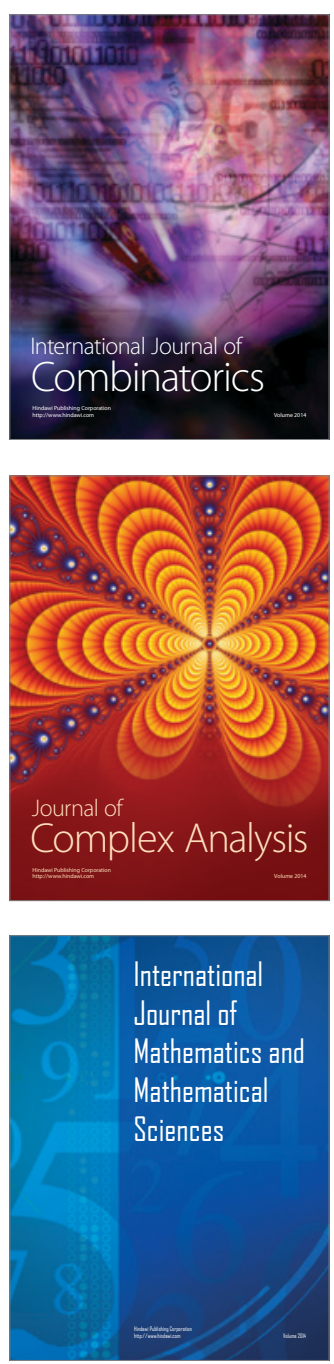
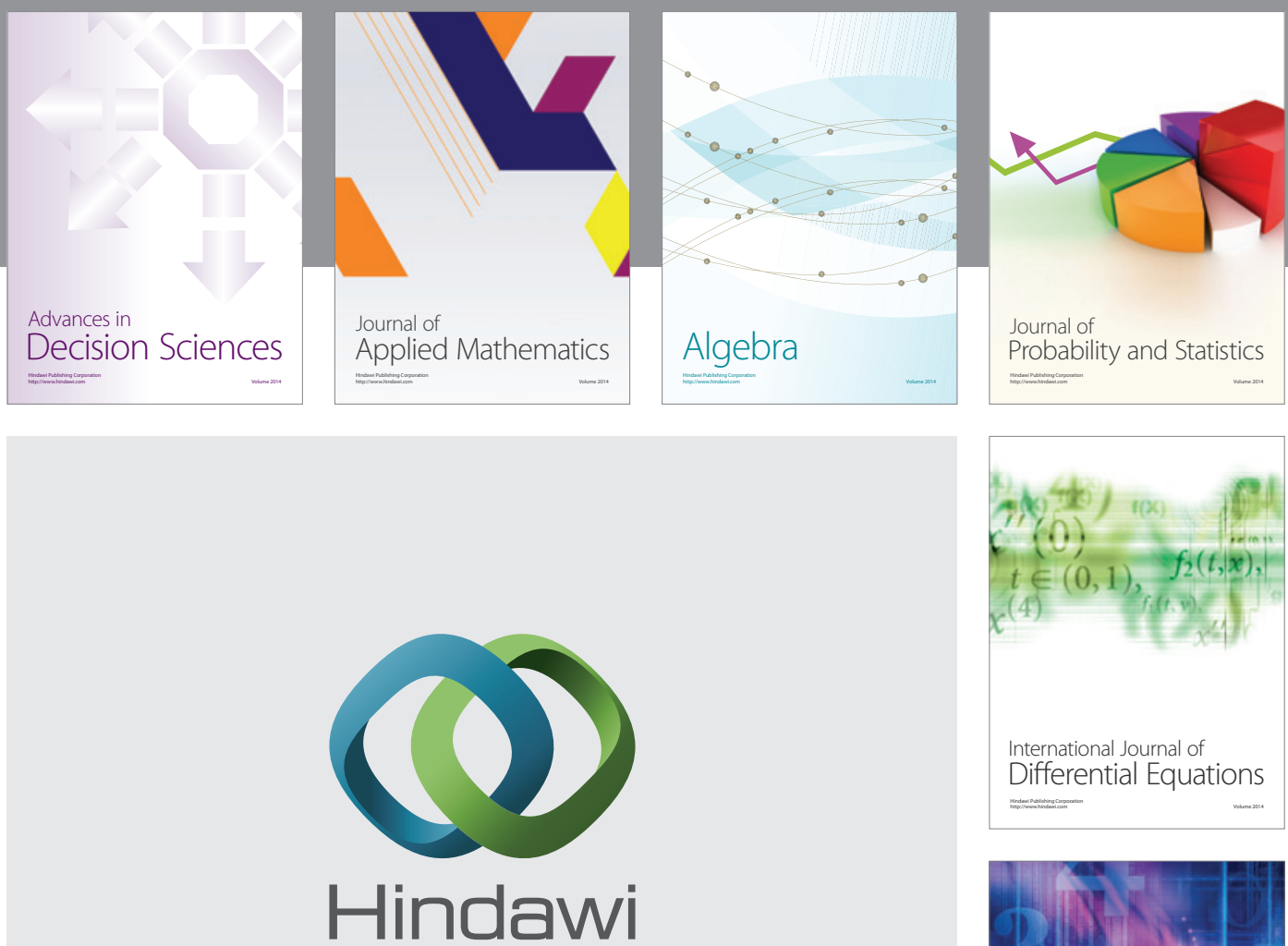

Submit your manuscripts at http://www.hindawi.com
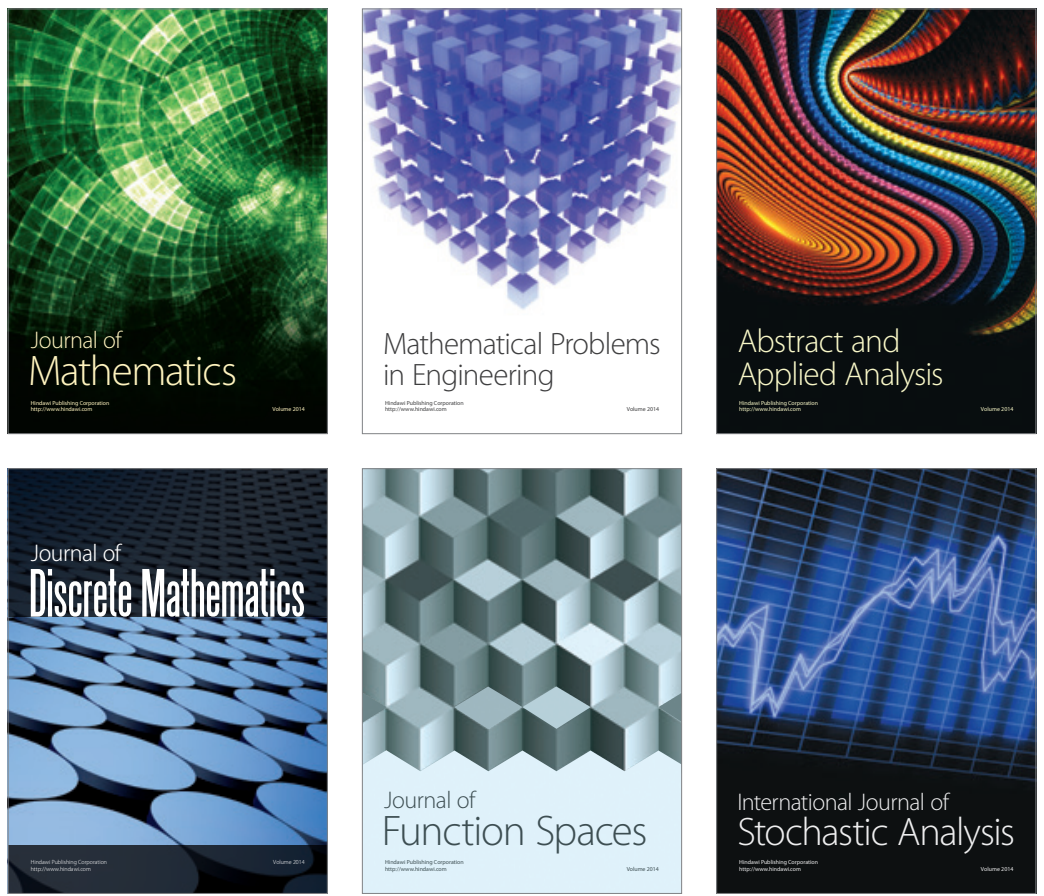

Journal of

Function Spaces

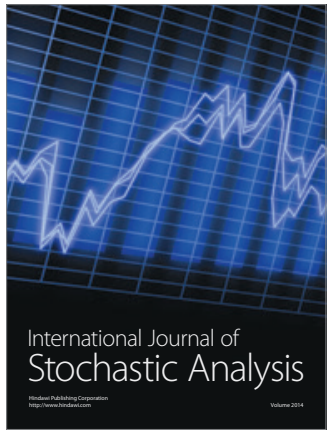

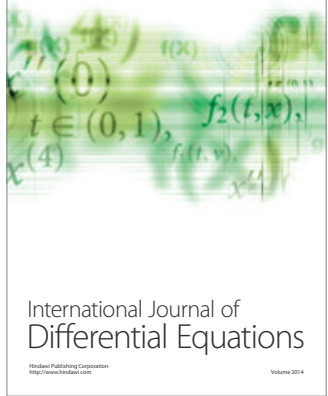
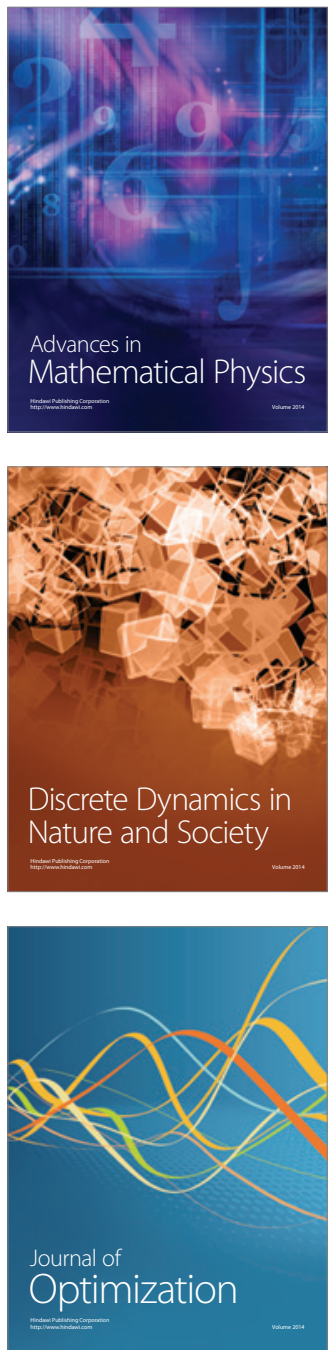